\title{
MEJORA DE LA CALIDAD, LA GESTIÓN Y LA DIFUSIÓN DE RELIEVE
}

\section{[Improvement of the quality, management, and circulation of RELIEVE]}

\author{
por
}

\begin{abstract}
$\underline{\text { Article record }}$
About authors

$\underline{\text { HTML format }}$
\end{abstract}

\author{
Correa, Ana D. (acorrea@ull.es)
}

Aliaga, Francisco M. (Francisco.Aliaga@uv.es) $\underline{\text { Ficha del artículo }}$

Sobre los autores

Formato HTML

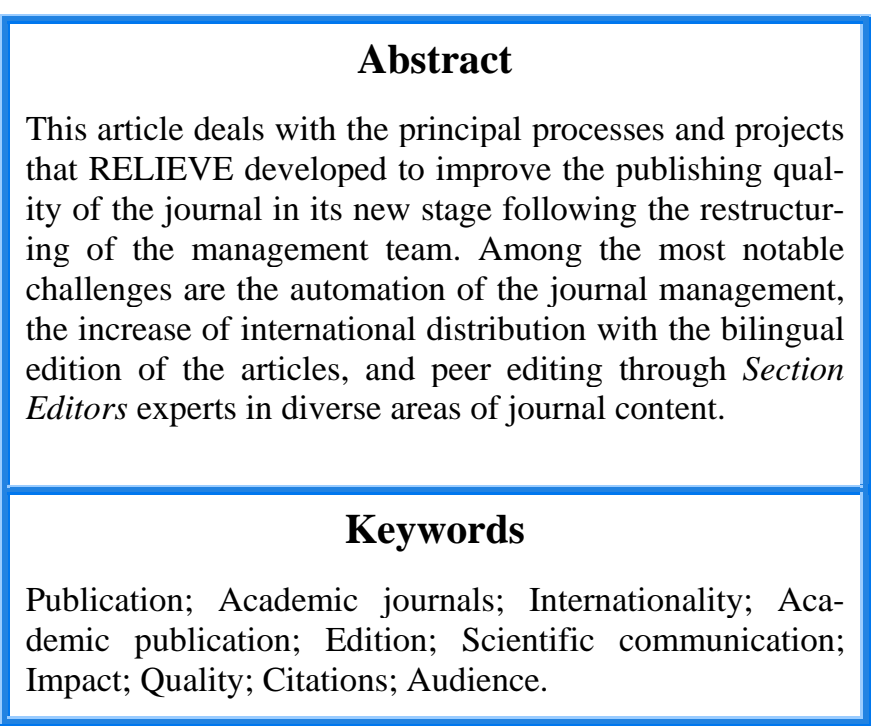

\begin{abstract}
Resumen
Se plantean los principales procesos y proyectos de RELIEVE planteados para la mejora de la calidad editorial de la revista en la nueva etapa, tras la reestructuración del equipo gestor. Entre los retos más relevantes destacan la automatización de la gestión de la revista, la ampliación de su difusión internacional con la edición bilingüe de los artículos y la edición colegiada mediante Editores de Sección expertos en los diversos ámbitos de contenido de la revista.

\section{Descriptores}

Publicación; Revistas académicas; Internacionalidad; Publicación académica; Edición; Comunicación científica; Impacto; Calidad; Citas; Audiencia.
\end{abstract}

En la última Asamblea General de la Asociación Interuniversitaria de Investigación Pedagógica (AIDIPE), celebrada en la Universidad de Huelva (España) en junio de 2009, se procedió a renovar la dirección de RELIEVE. El profesor Jesús Suárez Rodríguez, cesa como director a petición propia y tras ocho años de fructífera labor encabezando al equipo que ha conseguido mantener esta publicación en unos altos niveles de calidad e impacto, como han puesto de manifiesto los estudios sobre su repercusión cien- tífica (Aliaga y Suárez, 2008). Su puesto como director ha pasado a ser ocupado por el hasta ahora director ejecutivo, Francisco Aliaga, lo que garantiza la necesaria continuidad del trabajo que se ha venido realizando. Por su parte, Ana Delia Correa, que ha venido colaborando en calidad de revisora y miembro del Consejo Asesor, pasa a ocupar el cargo de directora ejecutiva ${ }^{[1]}$

RELIEVE ya ha cumplido tres lustros de existencia desde su pionero y, por ello, complicado inicio (Rodríguez Gómez, 2005). En 
este periodo ha sido gestionada por dos equipos directivos diferentes, por lo que este nuevo cambio (parcial) ya no es una prueba para su estabilidad, suficientemente consolidada. Es más bien una oportunidad para dar un nuevo impulso a la publicación, a sus procedimientos, su difusión y su calidad. Con esta finalidad en esta nueva etapa que se inicia, varios son los proyectos que estamos esbozando, con el propósito fundamental de seguir mejorando RELIEVE como instrumento de comunicación al servicio de la educación. La experiencia acumulada por los equipos anteriores nos permite al nuevo centrar la atención en los retos más relevantes. De forma similar, en la Revista de Investiga- ción Educativa, que también edita AIDIPE, Marín (2009) ofrece un conjunto de sugerencias de acción futura asentadas en la experiencia acumulada durante 26 años en la edición de dicha revista.

Uno de nuestros objetivos inmediatos es el de agilizar la gestión de la revista. El crecimiento continuo y acelerado que está disfrutando (y padeciendo) RELIEVE aconsejan cambiar los antiguos sistemas de gestión de originales por otro sistema más potente y eficiente. Como podemos observar en el Gráfico 1 , los originales recibidos en la redacción se han duplicado en sólo dos años.

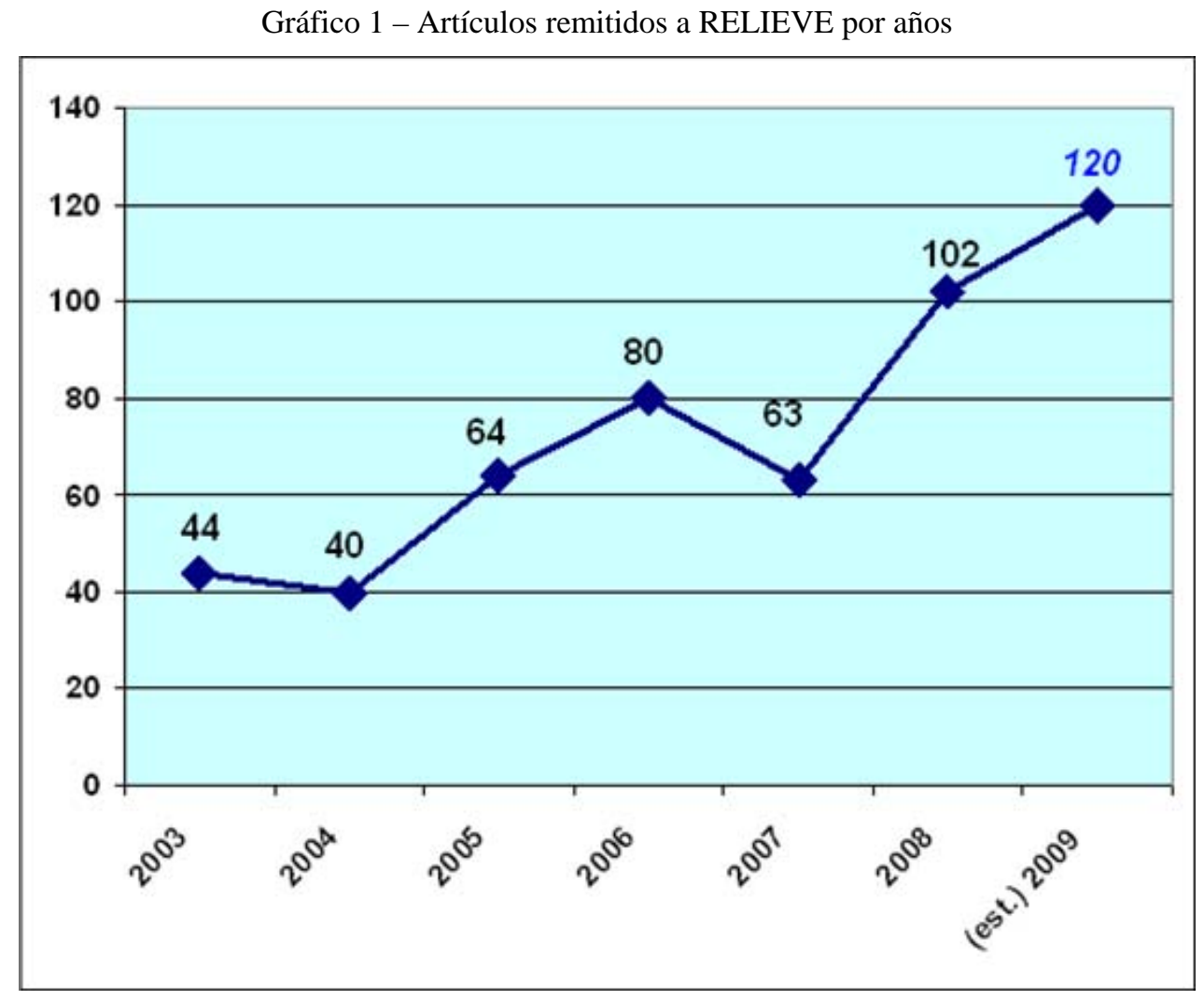

Los datos de 2009 son una estimación realizada con los datos disponibles a 1 de Noviembre

Para esta actualización aprovecharemos las ventajas derivadas de la inclusión en la plataforma RECyT (Repositorio Español de Ciencia y Tecnología). Esta inclusión ha sido posible gracias a que RELIEVE ha sido una de las revistas que ha superado con éxito la Primera evaluación de la Calidad de Revis- tas Científicas Españolas llevada a cabo por FECYT (Fundación Española de Ciencia y Tecnología), que es la responsable del repositorio, tras la convocatoria abierta en octubre de 2007. En dicho repositorio se halla integrado el programa de código libre Open Journal Systems (OJS) ${ }^{[2]}$, una potente herramienta de edición científica. RECyT cuenta 
con una Hemeroteca, que aloja un cierto número de revistas, y con un módulo de Edición de Revistas, que permite gestionar diversos aspectos como el envío de originales, la comunicación con autores, el proceso de revisión por pares, la corrección de estilo, el maquetado, etc. Esperamos que este sistema, aún en fase de adaptación y ensayo por nuestra parte, permita abreviar los tiempos que exige el procesado de los artículos, con el consiguiente beneficio para los autores y para la propia edición de la revista. En cualquier caso, esto afectará en principio sólo a la gestión de la revista, ya que los números seguirán publicándose en la Web habitual de RELIEVE. Aunque el uso de la plataforma es bastante sencillo e intuitivo, queremos facilitarlo en los momentos iniciales con un breve manual de instrucciones que está en preparación y que podrá consultarse desde el apartado de instrucciones para autores.

Extender nuestro alcance a un público más amplio es otra de nuestras pretensiones. Hasta el momento, RELIEVE ha tenido eco sobre todo (aunque no de manera exclusiva) en países de habla hispana, como se puso de manifiesto en el estudio sobre la internacionalidad de la revista hecho por Aliaga y Suárez (2007). Sin renunciar a seguir ampliando nuestra ya considerable presencia en Latinoamérica, queremos también estimular su alcance a otras audiencias. Una de las medidas que queremos adoptar con este fin es el incremento de la edición de artículos en inglés. En ocasiones, hemos publicado trabajos en este idioma, y un buen ejemplo de ello es la presencia de varios de tales artículos en este último número de 2009. También se viene procurando la traducción al inglés de aquellos artículos que alcanzan mayor difusión. El propósito es intentar que todos los artículos publicados en RELIEVE se editen en ambas lenguas. Esto supone pedir un poco más de esfuerzo a nuestros autores, tanto a hispanoparlantes como angloparlantes, pero entendemos que el potencial aumento de la repercusión y difusión que tendría su trabajo les compensará ampliamente. Ampliar la visibilidad de nuestra revista facilitando el acceso a sus contenidos a una audiencia más amplia (más allá de la concisa información que pueda aportar un Resumen o un Abstract) contribuirá a la lectura y, por tanto, citación de los trabajos que en ella se publiquen. Los autores que hayan consultado recientemente nuestras normas de edición habrán encontrado la recomendación añadida hace poco- de hacernos llegar la versión en inglés de los trabajos que ya hayan sido aceptados para su publicación.

Otro frente de acción encaminado a favorecer la expansión internacional de RELIEVE, es el de seguir gestionando su inclusión en más bases de datos y hemerotecas, elementos clave de difusión en los que el equipo anterior ya había obtenido grandes logros, que han supuesto que RELIEVE esté actualmente indexada en un gran número de bases de datos, como puede comprobarse en Aliaga y Suárez (2008). En este último año hemos seguido intensificando este proceso de inclusión en más bases de datos, tanto como medio de difusión del trabajo de nuestros autores como marchamo de calidad al superar los requisitos exigidos para el ingreso. En este sentido, la última incorporación conseguida hasta ahora se produjo en el primer semestre de 2009, y fue nada menos que la aceptación de RELIEVE en la prestigiosa SCOPUS. Esta base de datos se define como "la mayor base de datos de resúmenes y citas de literatura de investigación y de fuentes Web de calidad". Incluye treinta y ocho millones de referencias procedentes de 18.000 revistas académicas con filtro de calidad (revisión por pares). Patrocinada por el grupo Elsevier, pretende convertirse en el principal competidor de las bases de datos conocidas como ISI-Thompson (Web of Science).

Un aumento de la difusión podría suponer, paradójicamente, un enlentecimiento aparente en el número de consultas directas a la revista, ya que nuestros artículos pueden consultarse cada vez en más sitios independientes de nuestros servidores (EBSCO, Re- 
dalyc, Novadors, etc.). Las consultas realizadas a las copias de nuestros artículos depositadas en esos lugares no estarían contempladas, evidentemente, en nuestras estadísticas de visitas. La amplia difusión proporcionada por Internet tiene como contrapartida la dispersión, aunque en nuestro caso no lo consideramos como una rémora, ya que ayuda a dar una mayor difusión a nuestra revista.

Otro factor en el mismo sentido es la distinción alcanzada por RELIEVE al ser designada como 'Revista Fuente' en In-RECS, la organización encargada de medir el índice de impacto de las revistas españolas de Ciencias Sociales. Esto significa que, tras un exigente análisis, se le ha considerado dentro del "núcleo de revistas científicas españolas de Ciencias Sociales más influyentes”, tal y como se indica en el procedimiento de realización de su base de datos ${ }^{[3]}$. Todo ello a pesar de que en nuestro ámbito concreto de especialidad hay unos hábitos de citación muy poco generosos, que dan un índice de citas a revistas propias de la especialidad muy menguado. Ello repercute, sin ninguna duda, en la capacidad de proyección de las revistas especializadas en investigación y evaluación educativa, que ya de por sí es un área mucho menor (en el número de investigadores que implica) que otras de su entorno. Dentro de los parámetros de excelencia científica, el nivel de impacto de las revistas es un requisito básico y la citación es el indicador evaluativo más aceptado en la comunidad científica, lo que se operativiza con la contabilización de las referencias bibliográficas a dicha revista incluidas al final de un artículo. Aparte de otros criterios de calidad, el índice de impacto es el factor más relevante en el establecimiento de un ranking sobre la importancia de las publicaciones. Conviene tener en cuenta que los índices utilizados, sobre todo en ámbitos limitados, son extremadamente sensibles (o volátiles, según el punto de vista): variaciones muy pequeñas en el número de citas puede suponer cambios notables en el ranking. Ha de considerarse que cuentan, prioritariamente, las citas reali- zadas a artículos publicados en los últimos dos o tres años (según las distintas bases de datos) en revistas reconocidas, por ejemplo, las que están en ISI o son 'revistas fuente' en In-RECS (pueden consultarse en http://ec3.ugr.es/in-recs/listado/Educacionfuente.htm).

La citación, por tanto, es una potente herramienta al alcance de todos para impulsar el reconocimiento de las revistas de nuestro ámbito y contribuirá a nuestro ajuste a patrones establecidos sobradamente en otros ámbitos científicos bien consolidados, cuya "cultura de publicación" consigue posicionar y mantener sus revistas entre las más citadas. Hacemos un llamamiento a la reflexión de la comunidad académica, pero también, desde luego, a la acción.

En este sentido, estamos realizando una base de datos sobre las citas recibidas por artículos publicados en nuestra revista, que puede beneficiarse enormemente de la colaboración de nuestros lectores, los cuales pueden hacernos llegar la información cuando citen a RELIEVE en los artículos que publiquen, o bien cuando la vean citada en los artículos que consulten

Finalmente, una tercera iniciativa que ya está en marcha ha sido la de empezar a gestionar de forma colegiada la edición de RELIEVE en aquellas tareas que hasta ahora venían siendo responsabilidad exclusiva del Director Ejecutivo (o Editor). Son dos los motivos que nos han aconsejado este sistema de funcionamiento. Uno es el número creciente de originales que nos llegan para su consideración, tal como se puso de manifiesto en el Gráfico 1. La gestión fluida de un volumen tal de trabajos comienza a hacerse difícil para las posibilidades de un único Editor. La ralentización de los procesos, y el ritmo irregular de edición que provocaría a partir de cierto punto, podrían llegar a tener una repercusión negativa para la valoración de la calidad de la publicación. El segundo motivo también responde a un afán de mejo- 
ra de calidad en otro sentido más sustantivo, y es el de buscar un apoyo especializado que permita una gestión más adecuada y una toma de decisiones más experta, imprescindible por la variedad temática de los artículos que recibimos. Para ello, estamos organizando un reducido grupo de Editores de Sección, que trabajarán en estrecha colaboración con nosotros en la gestión de los artículos que por su contenido afectan a los ámbitos (secciones) de su competencia. La participación de los Editores de Sección, tanto en relación a su intercambio con la Dirección de la revista, como con los Autores y con los Revisores, son elementos previstos en las rutinas del programa OJS. En este sentido se han sumado ya al equipo directivo de RELIEVE como Editores de Sección Maria Teresa Aguado (UNED), J. Francisco Lukas (Universidad del País Vasco), Javier Gil (Universidad de Sevilla) y Mari Paz Sandín (Universidad de Barcelona).

En otro orden de cosas, desde el punto de vista técnico se ha producido una depuración de los contadores de visitas a algunos artículos. Por un problema en la programación de los mismos, algunos contadores se habían disparado a cifras poco razonables. Por fortuna contamos con una doble contabilidad independiente de las visitas, por lo que hemos podido identificar y corregir el problema. Con ello conseguiremos unos datos más fiables, tanto como indicador para los lectores (que pueden encontrar los artículos de más 'éxito') como para los propios autores y editores, que pueden realizar (y acreditar) esa difusión.

Otras tareas pendientes para un futuro cercano tienen que ver con la mejora en la utilización de palabras clave, tal y como se vienen planteando otras publicaciones (Sánchez Valle, 2005, 2007), en el que se exploraban las características de indexación de varias revistas, con el objeto de hacer propuestas para optimizarla. Esta es una cuestión clave, ya que los índices de visibilidad e impacto de las publicaciones guardan relación con la forma en que se alimentan las bases de datos, con el almacenamiento, recuperación y difusión de la información.

Es mucho lo que queda por hacer. Pero gracias al trabajo de los equipos editoriales anteriores podemos ver el camino que nos queda por recorrer desde una posición desahogada. Confiamos en que el nuevo impulso que venimos dispuestos a realizar siga permitiendo alcanzar las mayores cotas de calidad, difusión e impacto. Por el bien de la educación, que es la tarea común de todos nosotros.

\section{Referencias}

Aliaga, Francisco M. y Suárez-Rodríguez, J.M. (2007). Internacionalidad de las revistas académicas: Estudio de caso con RELIEVE. RELIEVE, v. 13, n. 1. http://www.uv.es/RELIEVE/v13n1/RELIE VEv13n1 0.htm. Consultado el 27/10/2009. Aliaga, Francisco M. y Suárez-Rodríguez, J. (2008). La repercusión científica de una revista académica: análisis del caso de RELIEVE. RELIEVE, v. 14, n. 2. http://www.uv.es/RELIEVE/v14n2/RELIE VEv14n2_0.htm. Consultado el 27/10/2009. Rodríguez Gómez, G. (2005). RELIEVE cumple 10 años, pero... ¿cómo fue el primero?. RELIEVE, v. 11, n. 2. http://www.uv.es/RELIEVE/v11n2/RELIE VEv11n2_1.htm. Consultado el 27/10/2009. Sánchez Valle, I. (2005). Análisis desde la perspectiva de la documentación científicopedagógica actual de la indización practicada en la revista Bordón (2001-2003). Bordón, v. 57, n 3, 379-396.

Sánchez Valle, I. (2007). Análisis de la indización practicada en la revista Pedagogía Social (1998-2003). Revista Complutense de Educación, Vol. 18, Núm. 1, 133-158.

Marín Gracia, M. A. (2009). Una revista para el siglo XXI. Mirando al futuro: una nueva etapa a partir de una sólida trayectoria. $R e$ vista de Investigación Educativa, v. 27, n.1, 3-5. 


\section{Notas}

[1] Desde aquí quiero agradecer el nombramiento a la Asociación, asumido con la preocupación de la mucha responsabilidad que implica el objetivo de mantener la revista en las cotas alcanzadas, pero con la confianza y seguridad que proporciona seguir contando con la demostrada experiencia de Cisco -en muchos sentidos el alma de RELIEVE-, con su apoyo sabio y generoso en estos momentos iniciales de andadura y aprendizaje y con la ayuda de todas las personas que hacen RELIEVE: Consejo Asesor, Consejo de Redacción, revisores y colaboradores (A.D. Correa).

[2] Open Journal System es una iniciativa de investigación y desarrollo del Public Knowledge Project de la University of British Columbia. http://pkp.sfu.ca.

[3] Ver http://ec3.ugr.es/inrecs/informacion/como1.htm\#3

\section{ABOUT THE AUTHORS / SOBRE LOS AUTORES}

Correa, Ana D. (acorrea@ull.es). Profesora Titular en el Departamento de Didáctica e Investigación Educativa de la Universidad de La Laguna (España). Su principal línea de trabajo es "Métodos de investigación". Su dirección postal es: Campus Central, Módulo B. C/ Delgado Barreto, s/n. 38071-La Laguna (Tenerife, España). Ha sido nombrada Directora Ejecutiva de RELIEVE en

Junio de 2009. Buscar otros artículos de esta autora en Scholar Google

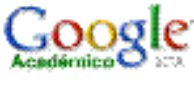

Aliaga, Francisco M. (Francisco.Aliaga@uv.es): Es Profesor Titular en el Departamento de Métodos de Investigación y Diagnóstico en Educación de la Universidad de Valencia (España), cuya dirección es Avda. Blasco Ibáñez, 30. 46010-Valencia (España). Sus principales líneas de trabajo son "Internet y Educación" y "Metodología de investigación". Fue Director Ejecutivo de RELIEVE desde 2001 hasta Junio de 2009, cuando ha sido promovido a Director. Buscar otros artículos $\underline{\text { de este autor en Scholar Google }}$ 


\section{ARTICLE RECORD / FICHA DEL ARTÍCULO}

\begin{tabular}{|c|c|}
\hline $\begin{array}{l}\text { Reference / } \\
\text { Referencia }\end{array}$ & $\begin{array}{l}\text { Correa, Ana D. y Aliaga, Francisco M. (2009). Mejora de la calidad, la gestión y la difusión de RELIEVE } \\
\text { RELIEVE, v. 15, n. 2. http://www.uv.es/RELIEVE/v15n2/RELIEVEv15n2_0.htm. }\end{array}$ \\
\hline Title / Título & $\begin{array}{l}\text { Mejora de la calidad, la gestión y la difusión de RELIEVE. [Improvement of the quality, management, and } \\
\text { circulation of RELIEVE]. }\end{array}$ \\
\hline $\begin{array}{l}\text { Authors / } \\
\text { Autores }\end{array}$ & Correa, Ana D. y Aliaga, Francisco M. \\
\hline $\begin{array}{l}\text { Review / } \\
\text { Revista }\end{array}$ & RELIEVE (Revista ELectrónica de Investigación y EValuación Educativa), v. 15, n. 2 \\
\hline ISSN & $1134-4032$ \\
\hline $\begin{array}{l}\text { Publication } \\
\text { date / } \\
\text { Fecha de } \\
\text { publicación }\end{array}$ & 2009 (Reception Date: 2008 November 9 [Editorial]; Publication Date: 2009 November 9). \\
\hline $\begin{array}{l}\text { Abstract / } \\
\text { Resumen }\end{array}$ & $\begin{array}{l}\text { This article deals with the principal processes and projects that RELIEVE developed to improve the pub- } \\
\text { lishing quality of the journal in its new stage following the restructuring of the management team. Among } \\
\text { the most notable challenges are the automation of the journal management, the increase of international } \\
\text { distribution with the bilingual edition of the articles, and peer editing through Section Editors experts in } \\
\text { diverse areas of journal content. } \\
\text { Se plantean los principales procesos y proyectos de RELIEVE planteados para la mejora de la calidad edi- } \\
\text { torial de la revista en la nueva etapa, tras la reestructuración del equipo gestor. Entre los retos más relevan- } \\
\text { tes destacan la automatización de la gestión de la revista, la ampliación de su difusión internacional con la } \\
\text { edición bilingüe de los artículos y la edición colegiada mediante Editores de Sección expertos en los diver- } \\
\text { sos ámbitos de contenido de la revista. }\end{array}$ \\
\hline $\begin{array}{l}\text { Keywords / } \\
\text { Descriptores }\end{array}$ & $\begin{array}{l}\text { Publication; Academic journals; Internationality; Academic publication; Edition; Scientific communica- } \\
\text { tion; Impact; Quality; Citations; Audience. } \\
\text { Publicación; Revistas académicas; Internacionalidad; Publicación académica; Edición; Comunicación } \\
\text { científica; Impacto; Calidad; Citas; Audiencia. }\end{array}$ \\
\hline $\begin{array}{l}\text { Institution / } \\
\text { Institución }\end{array}$ & Universidad de La Laguna y Universidad de Valencia (España). \\
\hline $\begin{array}{l}\text { Publication site } \\
\text { / Dirección }\end{array}$ & http://www.uv.es/RELIEVE \\
\hline $\begin{array}{l}\text { Language / } \\
\text { Idioma }\end{array}$ & Español \& English (Title, abstract and keywords in English \& Spanish) \\
\hline
\end{tabular}

\section{RELIEVE}

Revista ELectrónica de Investigación y EValuación Educativa E-Journal of Educational Research, Assessment and Evaluation

[ ISSN: 1134-4032 ]

(C) Copyright, RELIEVE. Reproduction and distribution of this articles it is authorized if the content is no modified and their origin is indicated (RELIEVE Journal, volume, number and electronic address of the document).

(C) Copyright, RELIEVE. Se autoriza la reproducción y distribución de este artículo siempre que no se modifique el contenido y se indique su origen (RELIEVE, volumen, número y dirección electrónica del documento). 